JOURNAL OF SYNCHROTRON RADIATION

ISSN 1600-5775

Received 16 April 2020

Accepted 2 September 2020

Edited by G. Grübel, HASYLAB at DESY, Germany

Keywords: X-ray photon correlation spectroscopy; refractive lenses.

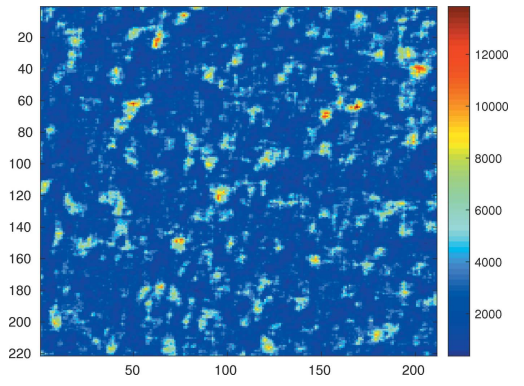

OPEN $\odot$ ACCESS

\section{Focusing a round coherent beam by spatial filtering the horizontal source}

\author{
Eric M. Dufresne, ${ }^{\mathrm{a} *}$ Suresh Narayanan, ${ }^{\mathrm{a}}$ Ruben Reininger, ${ }^{\mathrm{a}}$ Alec R. Sandy ${ }^{\mathrm{a}}$ and \\ Larry Lurio ${ }^{\mathbf{b}}$ \\ ${ }^{\mathbf{a}}$ Advanced Photon Source, Argonne National Laboratory, Argonne, IL 60439, USA, and ${ }^{\mathbf{b}}$ Department of Physics,
Northern Illinois University, DeKalb, IL, USA. *Correspondence e-mail: dufresne@anl.gov
}

This paper illustrates the use of spatial filtering with a horizontal slit near the source to enlarge the horizontal coherence in an experimental station and produce a diffraction-limited round focus at an insertion device beamline for $\mathrm{X}$-ray photon correlation spectroscopy experiments. Simple expressions are provided to guide the optical layout, and wave propagation simulations confirm their applicability. The two-dimensional focusing performance of Be compound refractive lenses to produce a round diffraction-limited focus at $11 \mathrm{keV}$ capable of generating a high-contrast speckle pattern of an aerogel sample is demonstrated. The coherent scattering patterns have comparable speckle sizes in both horizontal and vertical directions. The focal spot sizes are consistent with hybrid ray-tracing calculations. Producing a two-dimensional focus on the sample can be helpful to resolve speckle patterns with modern pixel array detectors with high visibility. This scheme has now been in use since 2019 for the 8-ID beamline at the Advanced Photon Source, sharing the undulator beam with two separate beamlines, 8-ID-E and 8-ID-I at $7.35 \mathrm{keV}$, with increased partially coherent flux, reduced horizontal spot sizes on samples, and good speckle contrast.

\section{Introduction}

Synchrotron light sources produce partially coherent light that has enabled new techniques such as X-ray photon correlation spectroscopy (XPCS) to flourish into mature material characterization methods (Sutton, 2008; Livet \& Sutton, 2012; Sinha et al., 2014; Sandy et al., 2018). Due to the large mismatch between the horizontal and vertical source size in third-generation synchrotron sources, which is typically a factor of 20 larger in the horizontal direction, the coherence length is a factor of 20 smaller in the horizontal than in the vertical direction. As a result, beamlines specializing in XPCS often focus the vertical coherent fan to match the smaller horizontal coherence length on the sample (Sandy et al., 2010; Chsuhkin, 2020).

Beamline 8-ID currently focuses the vertical coherent flux with a $1 \mathrm{D}$ parabolic Be lens. It is done at both branches, the 8-ID-I main line which specializes in small-angle XPCS, and the side branch 8-ID-E which focuses on wide-angle XPCS. One typically focuses the $125 \mu \mathrm{m}$ vertical coherent beam on the sample to a few $\mu \mathrm{m}$. The horizontal coherence is typically spatially filtered by a horizontal aperture set between 10 and $20 \mu \mathrm{m}$, placed 0.2 to $1.5 \mathrm{~m}$ before the sample, with the ranges given to show the typical openings and distances for the $\mathrm{E}$ and I branch, respectively. This optical scheme leads to different beam sizes on the sample and as a result different speckle sizes on the detector (Sutton, 2008). 
For some experiments on beamline 8-ID, one would like to use a $2 \mathrm{D}$ focusing compound refractive lens (CRL) with similar focal length as the existing 1-ID lens to produce a beam that better matches the micro-structure of materials. Focusing on a small spot size also produces larger speckle sizes which can be better suited for a large-pixel pixel array detector (PAD) with typical pixel size of $0.1 \mathrm{~mm}$. One would like to illuminate the sample with a 2D focused coherent beam, so, to achieve this, naively, one would reduce the horizontal beam size in the upstream hutch 8-ID-A with a slit to illuminate the lens coherently.

By choosing an appropriately small size of the horizontal slit, the diffracted beam will fill the lens with transversely coherent light. The flux will be reduced, but with the proper slit the optics will produce a diffraction-limited spot size.

We note that using a slit as a spatial filter is a well known technique at synchrotrons. It is used on the X-ray Nanoprobe for example at the APS (Winarski et al., 2012). On that beamline, a horizontal mirror focuses the horizontal fan of the source onto a beam-defining aperture that filters a coherent fan of light for the X-ray optics. This aperture produces a secondary horizontal source and should be placed as close as possible to the source. It enables the production of round beams on the sample after focusing with a short focal length zone plate.

Experimenters at the Petra III beamline P10 routinely use two-dimensionally focused beams in coherent diffraction experiments (Zozulya et al., 2012). Due to the very small horizontal source size at this facility, one can focus horizontally $50-100 \mu \mathrm{m}$ beams coherently to a few $\mu \mathrm{m}$ on the sample without closing any near front-end horizontal slit. This type of source spatial filtering allows one to deliver round focused coherent beams at existing synchrotrons with modest horizontal transverse coherent lengths $(10 \mu \mathrm{m}$ or below $)$.

Recently, several authors have modeled the propagation of coherence through optical systems including refractive lenses (Singer \& Vartanyants, 2014; Schroer \& Falkenberg, 2014). The first paper provides an analytical treatment which includes a slit placed just upstream of the lens and shows its impact to control the focal coherence length and spot size (Singer \& Vartanyants, 2014). This can be applied to the vertical focusing direction in this paper, although it approximates the slit by a Gaussian aperture. Since it does not include a treatment adding the horizontal secondary slit, we choose to simulate our results with advanced ray-tracing simulations.

Several ray-tracing approaches have been developed which include the propagation of coherence through optical elements from a synchrotron (Chubar et al., 2013; Shi et al., 2014; Wiegart et al., 2019). The work of Wiegart et al. (2019) is noteworthy for comparing measured coherent diffraction patterns from a thin grating sample illuminated by the 11-ID beamline of the National Synchrotron Light Source with simulations from the Synchrotron Radiation Workshop software (Chubar et al., 2013). The paper also describes the optical scheme of the beamline which focuses the beam in two dimensions with a vertical transfocator upstream in the optics hutch and a horizontal kineform lens in the experimental hutch (Wiegart et al., 2019).

In this paper we present a demonstration of $2 \mathrm{D}$ focusing with a long-focal-length Be lens to produce a round beam. We report the speckle size and contrast measured with focused coherent light. We present also optical simulations performed with a hybrid coherent diffraction module (Shi et al., 2014).

\section{Optical modeling}

The basic concept is to use a slit as a coherence filter in the horizontal direction so that its diffraction pattern in the lens plane matches the vertical transverse coherence length at the lens location. The lens can then be coherently illuminated by placing just upstream of the lens a square aperture with an opening equal to the vertical transverse coherence length

$$
l_{y}=\lambda R_{1} /\left(2 \sqrt{\pi} \Sigma_{y}\right),
$$

where $\Sigma_{y}$ is the root-mean-square (RMS) vertical source size, and $R_{1}$ is the distance source-lens (Dierker, 1997; Dufresne et al., 2002). The distance $R_{1}$ is shown in Fig. 1 . The factor $2 \sqrt{\pi}$ comes from the definition of the transverse coherence length for a Gaussian incoherent source from the Van CittertZernicke theorem (Goodman, 1985; Dierker, 1997).

Then, a horizontal slit placed upstream of the lens with an opening $\Delta$ will produce a diffraction pattern with a full width at half-maximum (FWHM) approximately equal to

$$
0.886 \lambda\left(R_{1}-R_{\mathrm{s}}\right) / \Delta=l_{y}
$$

where $R_{\mathrm{s}}$ is the distance from the source to the upstream horizontal slit. The factor 0.886 comes from the FWHM of the Fraunhofer diffraction pattern of a 1D aperture with opening $\Delta$. If one assumes that the horizontal source is spatially uniform at the white-beam slit and incoherent, the horizontal transverse coherence length at the lens is

$$
l_{x}=\lambda\left(R_{1}-R_{\mathrm{s}}\right) / \Delta,
$$

using the definition found in Goodman (1985).

Assuming that the undulator source is Gaussian in spatial and angular emission, the RMS horizontal beam size at a distance $R_{\mathrm{s}}$ from the source is approximately equal to $\left(R_{\mathrm{s}}^{2} \Sigma_{x^{\prime}}^{2}+\Sigma_{x}^{2}\right)^{1 / 2}$, where $\Sigma_{x^{\prime}}$ is the source horizontal divergence and $\Sigma_{x}$ the source size. The X-ray source divergence is

$$
\Sigma_{x^{\prime}}=\left(\sigma_{r^{\prime}}^{2}+\sigma_{\mathrm{ex}}^{2}\right)^{1 / 2}
$$

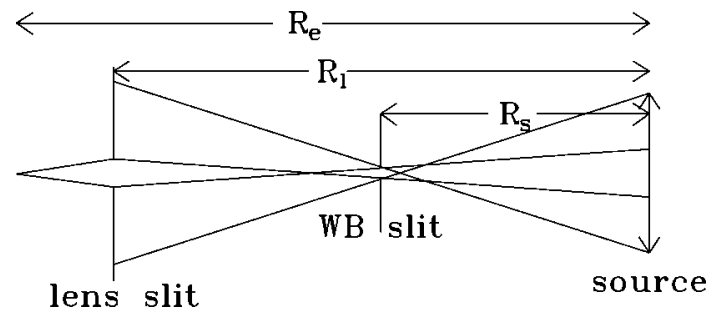

Figure 1

The horizontal rays collimation geometry. 
Table 1

Experimental parameters.

\begin{tabular}{lll}
\hline X-ray energy & $E$ & $10.6 \mathrm{keV}$ \\
RMS electron beam horizontal source size & $\sigma_{\mathrm{e} x}$ & $0.275 \mathrm{~mm}$ \\
RMS electron beam horizontal source divergence & $\sigma_{\mathrm{e} x^{\prime}}$ & $12.6 \mu \mathrm{rad}$ \\
RMS electron beam vertical source size & $\sigma_{\mathrm{e} y}$ & $0.010 \mathrm{~mm}$ \\
RMS electron beam vertical source divergence & $\sigma_{\mathrm{e} y^{\prime}}$ & $3.3 \mu \mathrm{rad}$ \\
Horizontal white-beam slit opening & $\Delta$ & $\sim 32 \mu \mathrm{m}$ \\
Source to white-beam slit distance & $R_{\mathrm{s}}$ & $27.0 \mathrm{~m}$ \\
2D lens location & $R_{\mathrm{l}}$ & $66.9 \mathrm{~m}$ from source \\
Source to experiment distance & $R_{\mathrm{e}}$ & $69.2 \mathrm{~m}$ \\
Vertical transverse coherence length at lens & $l_{y}$ & $207 \mu \mathrm{m}$ \\
Horizontal transverse coherence length at lens & $l_{x}$ & $8 \mu \mathrm{m}$ \\
Horizontal transverse coherence length at white-beam slit & $l_{x}$ & $3.3 \mu \mathrm{m}$ \\
Be lens radius of curvature & $R$ & $0.2 \mathrm{~mm}$ \\
Number of Be lenslet & $N$ & 16 \\
Dead layer of lenslet & $d_{0}$ & $\sim 50 \mu \mathrm{m}$ \\
2D aperture upstream of lens & $\left(\Delta_{\mathrm{l}}\right)^{2}$ & $0.125 \mathrm{~mm}$ by $0.125 \mathrm{~mm}$ \\
Sample to lens distance & $R_{\mathrm{e}}-R_{\mathrm{l}}$ & $2.29 \mathrm{~m}$ \\
\hline
\end{tabular}

where $\sigma_{r^{\prime}}=(0.5 \lambda / L)^{1 / 2}$ is the single electron source divergence through an undulator of length $L$, and $\sigma_{\mathrm{ex}}$ is the electron bunch RMS divergence. The X-ray vertical source size,

$$
\Sigma_{y}=\left(\sigma_{r}^{2}+\sigma_{\mathrm{ey}}^{2}\right)^{1 / 2},
$$

is the convolution of the single electron source size $\sigma_{r}=$ $(2 \lambda L)^{1 / 2} /(2 \pi)$ and the RMS electron beam size $\sigma_{\mathrm{ey}}$, so there is a $6 \%$ correction for hard X-rays of $1 \AA$ for a $L=2.4 \mathrm{~m}$-long undulator (Elleaume, 2002). A similar equation to equation (5) can be written in the horizontal direction for $\Sigma_{x}$, but the energy dependence is negligible due to the much larger electron beam size $\sigma_{\mathrm{e} x}$, thus $\Sigma_{x} \simeq \sigma_{\mathrm{e} x}$.

We note that the flux through the aperture is proportional to $\Delta / R_{\mathrm{s}}$, when $R_{\mathrm{s}} \Sigma_{x^{\prime}} \gg \Sigma_{x}$. The horizontal beam size at the APS becomes dominated by the horizontal divergence for distances larger than $\Sigma_{x} / \Sigma_{x^{\prime}} \simeq 22 \mathrm{~m}$ (see Table 1), which is upstream of the front-end.

Putting equation (1) into equation (2), one finds

$$
\Delta \simeq 3.14 \Sigma_{y} \frac{\left(R_{1}-R_{\mathrm{s}}\right)}{R_{\mathrm{l}}} .
$$

It is fairly independent of wavelength if one neglects the small energy dependence of the source size. The throughput from slitting the horizontal can be approximated by

$$
\phi=\frac{\Delta}{\sqrt{2 \pi}\left(R_{\mathrm{s}}^{2} \Sigma_{x^{\prime}}^{2}+\Sigma_{x}^{2}\right)^{1 / 2}},
$$

where $\phi$ is the partially coherent flux transmitted through the slit, assuming the slit opening $\Delta$ is much smaller than the beam profile at the slit position. By inspection of equation (7), $\Delta$ is largest when $R_{\mathrm{s}}=0$, and the beam size is also the smallest, thus the defining slit should be as close as possible to the source to optimize the flux. At the APS, white-beam slits are located after the front-end, but at some synchrotrons they are accessible before the front-end (Wiegart et al., 2019). Reducing the horizontal beam also reduces the power on X-ray optics downstream of the slit.

At the APS, the RMS horizontal and vertical electron beam size are, respectively, $\sigma_{\mathrm{e} x}=0.275 \mathrm{~mm}$ and $\sigma_{\mathrm{e} y} \simeq 0.010 \mathrm{~mm}$, so they differ by a factor of 27 . For a ratio $R_{\mathrm{s}} / R_{1} \simeq 1 / 2$, the small opening $\Delta$ will reduce the source divergence such that the main contribution is the pinhole camera of the source through the aperture $\Sigma_{x^{\prime}} \simeq \Sigma_{x} / R_{\mathrm{s}}$, thus for $R_{\mathrm{s}}=27 \mathrm{~m}$, $\Sigma_{x^{\prime}}=10 \mu \mathrm{rad}$. We note that, from equation (4), $\Sigma_{x^{\prime}} \simeq 13.5 \mu \mathrm{rad}$ at $11 \mathrm{keV}$.

The RMS beam size at the lens position will be the convolution of the diffraction pattern of the slit $\Delta$ and beam size propagated through the aperture. Assuming that the source irradiance is both Gaussian in space and angular emission, one can show that the RMS beam profile at the lens should be written as

$$
\begin{aligned}
\Sigma_{x l}= & \left\{\frac{1}{R_{\mathrm{s}}^{2} /\left[\left(R_{\mathrm{l}}-R_{\mathrm{s}}\right)^{2} \Sigma_{x}^{2}\right]+1 /\left[\left(R_{\mathrm{l}}-R_{\mathrm{s}}\right)^{2} \Sigma_{x^{\prime}}^{2}\right]}\right. \\
& \left.+\left[0.377 \lambda\left(R_{\mathrm{l}}-R_{\mathrm{s}}\right) / \Delta\right]^{2}\right\}^{1 / 2} .
\end{aligned}
$$

The first term is derived in Appendix $A$ and comes from the pinhole camera image of the horizontal source with finite source size and divergence. The second term on the sum in equation (8) is the width of the diffraction pattern of the slit with opening $\Delta$. We note that an exact treatment is discussed for a bending magnet pinhole camera diagnostic (Yang et al., 2017) including Fresnel diffraction. This is not needed as we are in the far-field of the slit.

Looking back at Fig. 1, one can show that the two slits form a collimator with collimation angle

$$
\theta=\left(\Delta+\Delta_{\mathrm{l}}\right) /\left(R_{\mathrm{l}}-R_{\mathrm{s}}\right),
$$

where $\Delta_{1}$ is the slit opening in front of the lens. This collimator limits the size of the source to a perceived horizontal size

$$
\Delta_{x}=\frac{R_{\mathrm{s}}\left(\Delta+\Delta_{\mathrm{l}}\right)}{R_{\mathrm{l}}-R_{\mathrm{s}}}+\Delta .
$$

Using values found in Table 1 for the distances, and whitebeam slit opening $\Delta$, while using $\Delta_{1}=0.125 \mathrm{~mm}$, one finds $\Delta_{x}=$ $0.138 \mathrm{~mm}$. Using equation (3), this collimation increases the source transverse coherence length at the white-beam slit to $l_{x}^{\prime}=\lambda R_{\mathrm{s}} / \Delta_{x}=23 \mu \mathrm{m}$ for values defined in Table 1 and used in this paragraph. In this example, $l_{x}^{\prime} / \Delta=71.5 \%$, which is an improvement from the original ratio $l_{x} / \Delta \simeq 10 \%$ in Table 1 .

The focal length of a parabolic compound refractive lens in the thin lens approximation is given by

$$
f=\frac{R}{2 N \delta},
$$

where $R$ is the radius of curvature of a lenslet, $N$ is the number of lenslets, and $\delta$ is the index of refraction decrement (Snigirev et al., 1996; Lengeler et al., 2002). When the lens can view the full source, if the source-lens distance $R_{1} \gg f$, we expect the RMS focal spot size to be a demagnified image of the source with $\sigma_{\mathrm{i}} \simeq f \Sigma_{\mathrm{s}} / R_{\mathrm{l}}$, where $\Sigma_{\mathrm{s}}$ is the RMS source size. 
If we use a small white-beam slit opening instead, then a rough estimate of the focal spot size is

$$
w=\left(w_{\mathrm{i}}^{2}+w_{\mathrm{d}}^{2}\right)^{1 / 2},
$$

where $w_{\mathrm{d}}=0.886 \lambda\left(R_{\mathrm{e}}-R_{\mathrm{l}}\right) / \Delta_{l}$, which is the FWHM of the diffraction pattern of the lens slit aperture at the focal distance $R_{\mathrm{e}}$ measured from the source. In equation (12), the demagnified source image width,

$$
w_{\mathrm{i}}= \begin{cases}\Delta\left(R_{\mathrm{e}}-R_{\mathrm{l}}\right) /\left(R_{\mathrm{l}}-R_{\mathrm{s}}\right) & \text { horizontally, or } \\ 2.35 \Sigma_{y}\left(R_{\mathrm{e}}-R_{\mathrm{l}}\right) / R_{\mathrm{l}} & \text { vertically. }\end{cases}
$$

In equation (13), we use the white-beam slit opening as the source for the demagnified horizontal source width. For values found in Table 1, we find the FWHM horizontal spot size $w=2.6 \mu \mathrm{m}$.

We define the depth of focus as the distance $\Delta z$ from the best focal plane such that the FWHM of the beam is

$$
W=\left[w_{\mathrm{m}}^{2}+\left(\frac{\Delta_{\mathrm{l}} \Delta z}{R_{\mathrm{e}}-R_{\mathrm{l}}}\right)^{2}\right]^{1 / 2}=\sqrt{2} w_{\mathrm{m}},
$$

where $w_{\mathrm{m}}$ is the computed focal spot in equation (12). One finds

$$
\Delta z= \pm w_{\mathrm{m}}\left(R_{\mathrm{e}}-R_{\mathrm{l}}\right) / \Delta_{\mathrm{l}} .
$$

Note that $w_{\mathrm{m}}$ depends on $\Delta_{\mathrm{l}}$, the slit opening before the lens. Using the vertical minimum focus, $w_{\mathrm{m}}=2.0 \mu \mathrm{m}$, the vertical depth of focus is $\Delta_{z}=3.7 \mathrm{~cm}$.

\section{X-ray optical simulations}

X-ray optical simulations were performed with Shadow using the Hybrid method reported by Shi et al. (2014). The X-ray energy was set to $11.0 \mathrm{keV}$ so that the focal distances would match the experimental setup described in Table 1 . The twodimensional aperture set to $0.125 \mathrm{~mm} \times 0.125 \mathrm{~mm}$ is placed $59 \mathrm{~cm}$ before the lens. Fig. 2 shows the simulation results in the plane with the best vertical focus of a 2D Be lens. The focal spot size in the horizontal and vertical direction is shown in the plot legend as well as the transmission of the optical system of $0.059 \%$. We note that clear diffraction effects are noticeable in the vertical direction due to the fact that the transverse coherence length vertically is larger than $\Delta_{1}=$ $0.125 \mathrm{~mm}$.

Equation (12) provides a reasonable estimate of the horizontal spot size (in the simulations here $2.9 \mu \mathrm{m}$ ) to be $2.6 \mu \mathrm{m}$ as discussed earlier. Using equation (12), and data from Table 1, we find the FWHM of the vertical spot size $w=2 \mu \mathrm{m}$, in excellent agreement with the simulations.

Fig. 3 shows the vertical spot size as a function of the longitudinal distance from the lens. The calculated distance from the lens using the thin lens formula is $2.29 \mathrm{~m}$ and corresponds to the vertical focal distance in the simulations. In these simulations, the source is assumed to be Gaussian and centered on the center of the 8-ID straight section.
Transmission: $0.059 \%$

FWHMx:2.9 $\mu \mathrm{m} \quad$ FWHMy: $2 \mu \mathrm{m}$

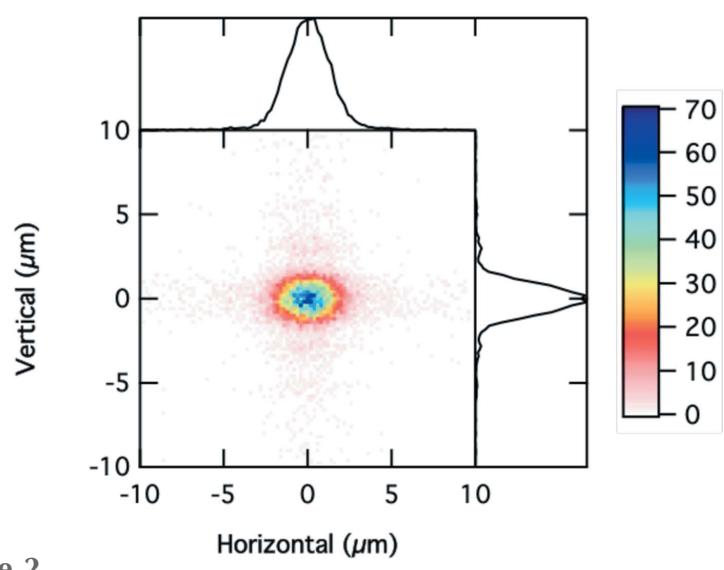

Figure 2

Simulated focal spot profile in 8-ID-I with a white-beam slit opening of $32 \mu \mathrm{m}, 2.29 \mathrm{~m}$ from the lens. The central part of the figure shows the intensity distribution. The two graphs above and at the right in the figure show the cumulative intensity in each direction. The color scale displays the intensity.

Fig. 4 shows the horizontal spot size as a function of the longitudinal distance from the lens. The horizontal focal plane is about $5 \mathrm{~cm}$ downstream from the vertical focal position. Using the thin lens formula with the horizontal source position at the white-beam slit location, we find the image distance from the lens should be $2.344 \mathrm{~m}$, consistent with the simulations.

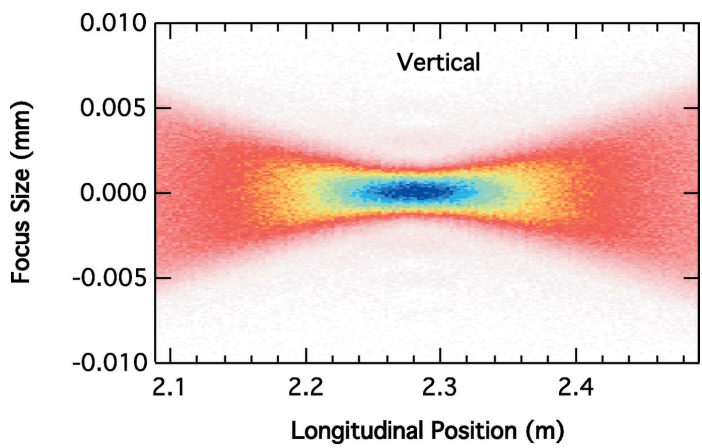

Figure 3

Simulated vertical focal spot profile in 8-ID-I as a function of the distance from the lens with a white-beam slit opening of $32 \mu \mathrm{m}$.

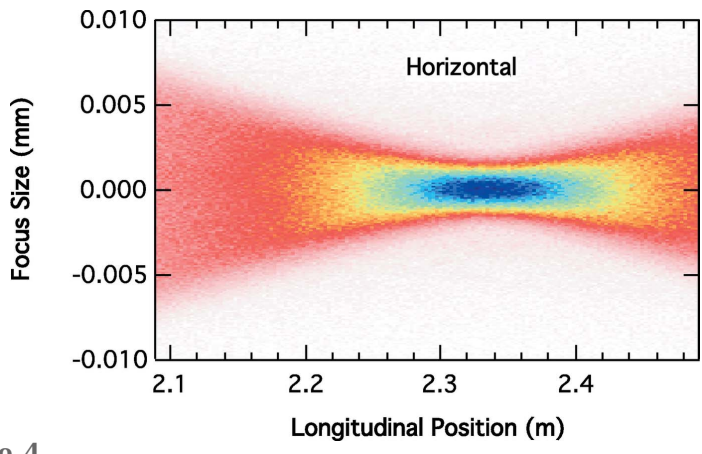

Figure 4

Simulated horizontal focal spot profile in 8-ID-I as a function of the distance from the lens with a white-beam slit opening of $32 \mu \mathrm{m}$. 
Transmission: $0.059 \%$

FWHMx:2.2 $\mu \mathrm{m} \quad$ FWHMy:2.8 $\mu \mathrm{m}$

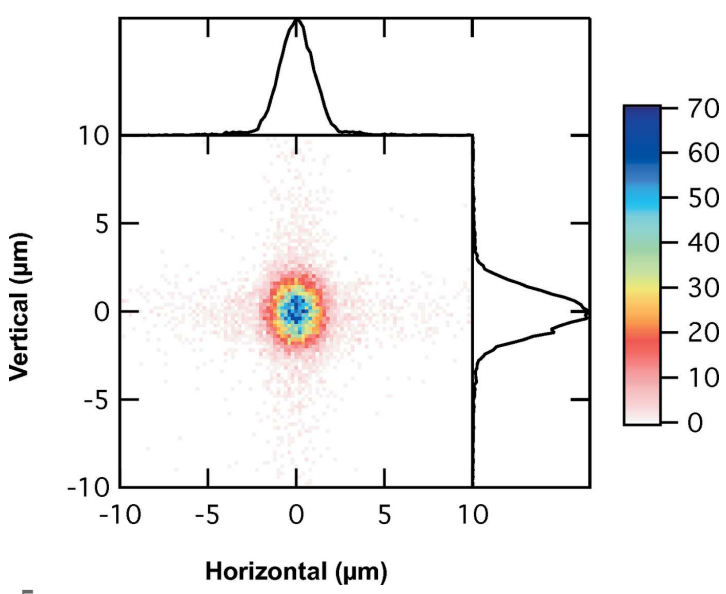

Figure 5

Simulated focal spot profile in 8-ID-I $2.34 \mathrm{~m}$ from the lens with a whitebeam slit opening of $32 \mu \mathrm{m}$ showing the smallest horizontal spot size.

Fig. 5 shows the focal spot at the best horizontal focus in Fig. 4. Because the horizontal and vertical source points are not in the same plane, it is not possible to optimize both spot sizes simultaneously. The focal area, i.e. the product of the vertical and horizontal FWHM beam sizes, only varies by $6 \%$ between the two planes defined in Figs. 2 and 5. The distance between the horizontal and vertical best focus, here $5 \mathrm{~cm}$, compares with the vertical depth of focus of $3.7 \mathrm{~cm}$ computed in equation (15), but both distances are much larger than a typical sample thickness $(\sim 3 \mathrm{~mm})$ used in our XPCS experiments, thus the astigmatic focus does not cause significant beam waist changes over the illuminated length. An experimenter has the choice to explore beam profile extremes shown in Figs. 2 and 5 by moving the sample along the beam direction.

Simulations were also performed using a $1 \mathrm{D}$, vertically focusing Be CRL. Fig. 6 shows the focal spot profile, with a

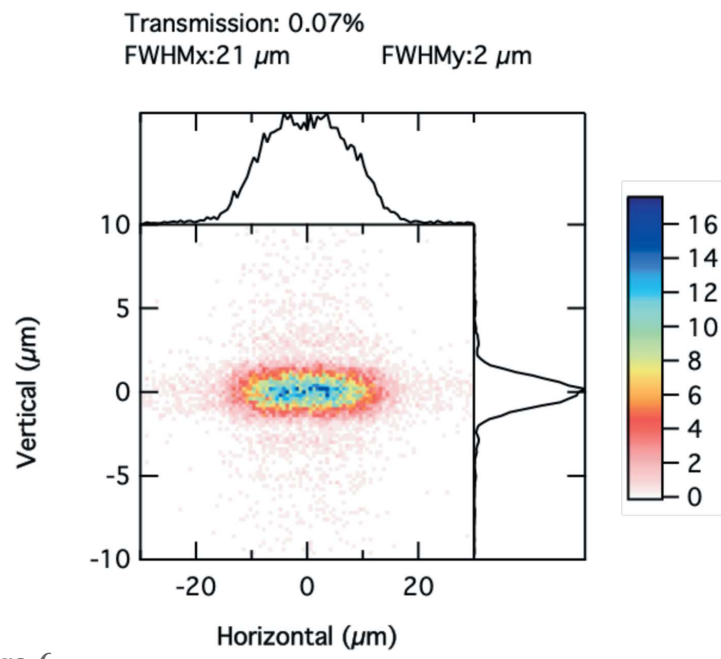

Figure 6

Simulated focal spot profile in 8-ID-I, using vertical focusing only with a wide-open white-beam slit opening. horizontal coherence slit set to $20 \mu \mathrm{m}$, placed $88 \mathrm{~cm}$ after the lens. The vertical focal spot has also a FWHM of $2.0 \mu \mathrm{m}$, while the horizontal profile is slightly broadened by Fraunhofer diffraction to a FWHM of $21 \mu \mathrm{m}$. The transmitted partially coherent flux is $0.069 \%$ of the total emitted flux, thus about $17 \%$ higher than for the 2D focusing case. The coherent flux transmitted through an aperture $l_{x} \times l_{y}$ at the lens position in Table 1 is $0.063 \%$ of the total emitted flux, as computed with the XUS undulator simulation tool in $X O P$. The optical simulations are performed with wider horizontal slits than the $X O P$ estimate, but they include geometrical and reflectivity losses so the transmitted flux are similar to the $X O P$ estimate.

\section{Experimental method}

The experiment was performed on beamline 8-ID-I of the APS. Two $33 \mathrm{~mm}$-period and $2.4 \mathrm{~m}$-long undulator A provide bright coherent X-rays on 8-ID. They add in phase at 7.35 and $11 \mathrm{keV}$. Our test used only the upstream undulator A. Following the front-end, a small $0.275 \mathrm{~mm}$-diameter watercooled pinhole located $26.0 \mathrm{~m}$ from the source reduces the incident power on the beamline optics to a few Watts (Sandy et al., 1999, 2010).

The white-beam slit is located downstream of the pinhole. Its opening can be estimated from equation (2), with $R_{1}=$ $66.9 \mathrm{~m}, R_{\mathrm{s}}=27 \mathrm{~m}, \lambda=1.127 \AA(11 \mathrm{keV})$, and FWHM $=$ $0.125 \mathrm{~mm}$. The FWHM used here is a typical slit opening we use in front of the lens, and is significantly smaller than the vertical transverse coherence length calculated from equation (1) and shown in Table 1 . We roughly set the whitebeam slit opening to $32 \mu \mathrm{m}$ but it was challenging due to mechanical issues with the slit stages. The tungsten edges are polished to $0.2 \mu \mathrm{m}$. The white beam apertured by the slit is reflected by a water-cooled Si mirror with a $2.5 \mathrm{mrad}$ angle of incidence. The slit reduces the illumination of the mirror to approximately $12 \%$ of its $11.0 \mathrm{~cm}$ length. A Ge (111) doublecrystal monochromator diffracting vertically was set to $10.6 \mathrm{keV}$ (Narayanan et al., 2008). Although we planned to work at $11 \mathrm{keV}$, the best focus ended up at $10.6 \mathrm{keV}$.

The focal length was adjusted by scanning the energy of the undulator and monochromator. The focal spot size was estimated by measuring the speckle size from an aerogel using a well known spatial autocorrelation technique (Sandy et al., 1999, 2010). We note that this technique was recently extended to characterize the focal spot size of CRL at the LCLS (Sikorski et al., 2015). The technique is capable of measuring the focal spot size in 2D with single-shot sensitivity, thus enabling X-ray laser shot-to-shot focus size monitoring. The focal spot size was measured by the transmitted intensity with a PIN diode while scanning a knife-edge using a cleaved GaAs wafer. These are well known to have atomically flat edges convenient to probe a micrometre focal spot, or to be used as slit blades (Dufresne et al., 2009). The X-ray flux reported in this paper was calculated from the measured photocurrent from the diode. 


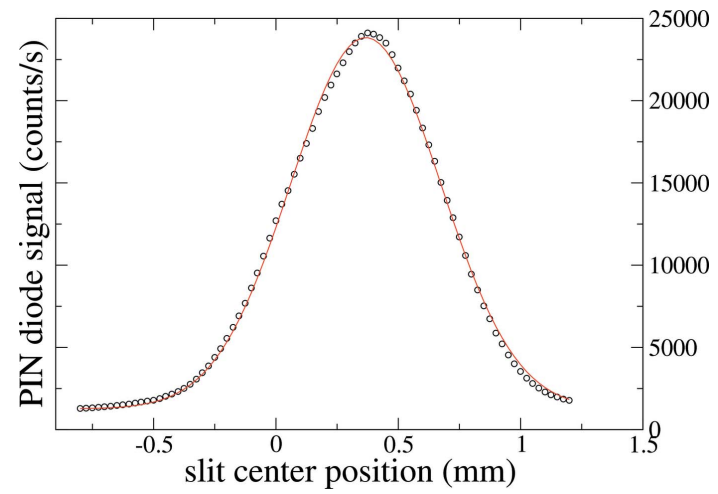

Figure 7

Horizontal beam profile in 8-ID-I just before the lens, with a white-beam slit opening of approximately $32 \mu \mathrm{m}$. The solid line is a Gaussian leastsquares fit described in the text.

\section{Results}

The horizontal beam profile in 8-ID-I upstream of the lens is shown in Fig. 7. It is measured with a $20 \mu \mathrm{m}$ horizontal lens entrance slit placed $0.584 \mathrm{~m}$ upstream of the lens (see Fig. 1). It is much larger than the diffraction pattern of the whitebeam slit because the white-beam slit acts as a pinhole camera as shown in Fig. 1. The measured FWHM of Fig. 7 is $0.757 \mathrm{~mm}$, while using equation (8) one finds a FWHM of $0.754 \mathrm{~mm}$, in excellent agreement with the data. A Gaussian least-squares fit is shown with a FWHM of $0.72 \mathrm{~mm}$, and a small background of 1270 counts $\mathrm{s}^{-1}$. We note also that the measured beam profile is slightly asymmetric which we attribute to the fact that the two blades of the white-beam slits do not reside in the same plane. This effect has been shown to create an angledependent opening in coherence slits (Libbert et al., 1997).

We chose the number of lenses $N$ such that it would focus $11 \mathrm{keV}$ X-rays in the usual 8-ID-I sample location. To ensure we achieved the best focus, we illuminated an aerogel sample with focused light and measured the speckle contrast and speckle size from a well known technique, discussed in Appendix $B$ and by Sandy et al. (2010).

Fig. 8 shows the speckle size as a function of the incident $\mathrm{X}$-ray energy in the horizontal and vertical direction. Since the speckle size is proportional to the width of the Fraunhofer diffraction pattern of the illuminated sample area, the speckle size should be maximum at the best focus energy. We note that the speckle size is larger in the vertical direction most likely due to the smaller vertical source size. The data also suggest a slight difference in focal length for the horizontal and vertical direction due to the difference in peak energy of the speckle size. Since $f$ increases with energy, the horizontal speckle size peak maximum suggests the horizontal focus is upstream of the vertical one, in contrast with the simulations.

It is possible that this effect is caused by slope errors on the water-cooled $\mathrm{Ge}(111)$ monochromator. We have found that for vertical focusing we need to add more lenses to focus at a given distance (Zhang et al., 2016). Zhang et al. vertically focused a $7.35 \mathrm{keV} X$-ray beam in 8 -ID-I using the same geometry with Be lenses with $R=0.2 \mathrm{~mm}$ using $N=10$ instead

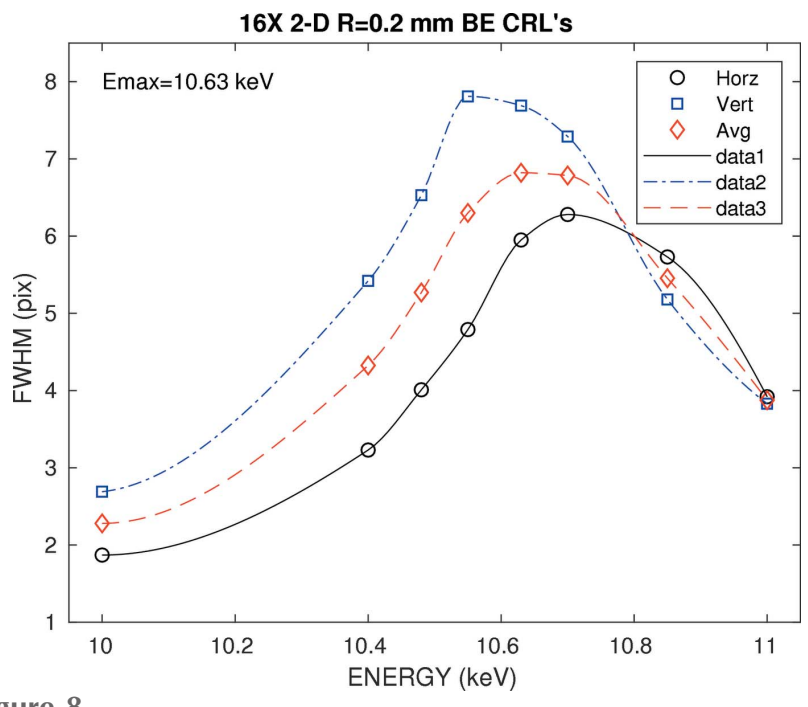

Figure 8

Measured horizontal and vertical speckle size from an aerogel pattern located in the focal plane. A pixel is $20 \mu \mathrm{m}$.

of $N=7$, the best choice from optical constants and equation (11) (Zhang et al., 2016). Calculations of this effect have been discussed recently (Antimonov et al., 2016).

Fig. 9 shows the speckle contrast as a function of incident energy. The contrast is maximum near $10.6 \mathrm{keV}$, but is not as sensitive to energy as the speckle size, and some small scatter is present near the peak energy. The maximum contrast is approximately $29 \%$, which is close to the typical contrast on 8 -ID-I in the unfocused condition.

In this experiment, we scanned the incident energy and doing so moved the focus longitudinally along the beam propagation direction. Given the quadratic energy dependence of $\delta(E) \propto 1 / E^{2}$, a $100 \mathrm{eV}$ shift reduction moved the focus upstream by $\Delta z=2 f \Delta E / E \simeq 4 \mathrm{~cm}$. It thus increased the illumination spot size by $\Delta_{1} / f \Delta z=2 \Delta E / E \Delta_{1} \simeq 2.3 \mu \mathrm{m}$. Thus we can also control the illuminated area by detuning the energy from the optimal focus. Changing the numerical aperture would also control the focal spot size.

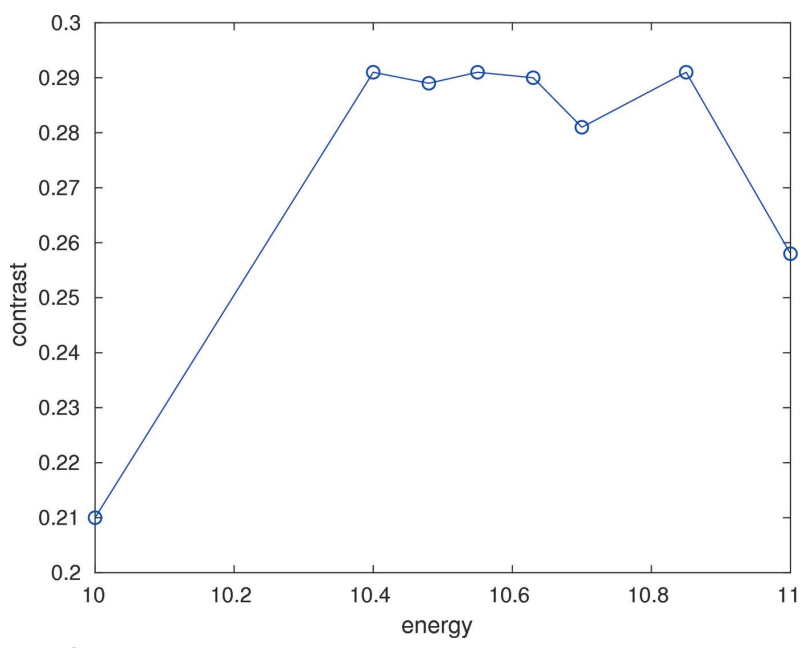

Figure 9

Measured speckle contrast from an aerogel pattern located in the focal plane. 
We chose to probe the focus with a knife-edge scan at $10.6 \mathrm{keV}$. Fig. 10 shows the results of the vertical knifeedge scan with a step size of $0.5 \mu \mathrm{m}$, after taking its spatial derivative. The vertical beam profile derived from the derivative has a FWHM of approximately $3 \mu \mathrm{m}$, and compares well with the simulations $(2 \mu \mathrm{m})$. The vertical profile shows a long asymmetric tail with a possible diffraction fringe from the $125 \mu \mathrm{m}$ slit. The fringe could be due in part to the increased transverse coherence in the vertical direction. Vertical fringes are visible in the simulation in Fig. 2.

Fig. 11 shows the focal spot size with a Gaussian and Lorentzian non-linear least-squared fit. The FWHM of the data is $3 \mu \mathrm{m}$. It compares well with estimates made earlier of $2.9 \mu \mathrm{m}$. The data overall lie between the two line shapes, indicating some tails with larger background than a Gaussian line shape. We note that the horizontal profile is significantly smaller than the FWHM of the demagnified source size $2.35 \sigma_{x}\left(R_{\mathrm{e}}-R_{1}\right) / R_{1}=22 \mu \mathrm{m}$. We achieved diffraction-limited focusing in the horizontal as well as in the vertical direction. This is in good qualitative agreement with the simulations performed.

The results were recently reproduced simultaneously on two beamlines 8-ID-E and 8-ID-I at the APS at $7.35 \mathrm{keV}$. The experimental conditions are shown in Table 2. For this experiment, both our inline undulators were closed to produce optimal flux at $7.35 \mathrm{keV}$. From equation (1), the vertical transverse coherence length at this energy is $318 \mu \mathrm{m}$ in 8 -ID-I, but we typically use $150 \mu \mathrm{m}$ aperture before the lens when we focus vertically, thus using equation (2) with $l_{y}=150 \mu \mathrm{m}$, we set the white-beam slit to $40 \mu \mathrm{m}$ horizontally, optimal for the 8-ID-I lens distance. A $\mathrm{Si}(220)$ crystal bisected the beam

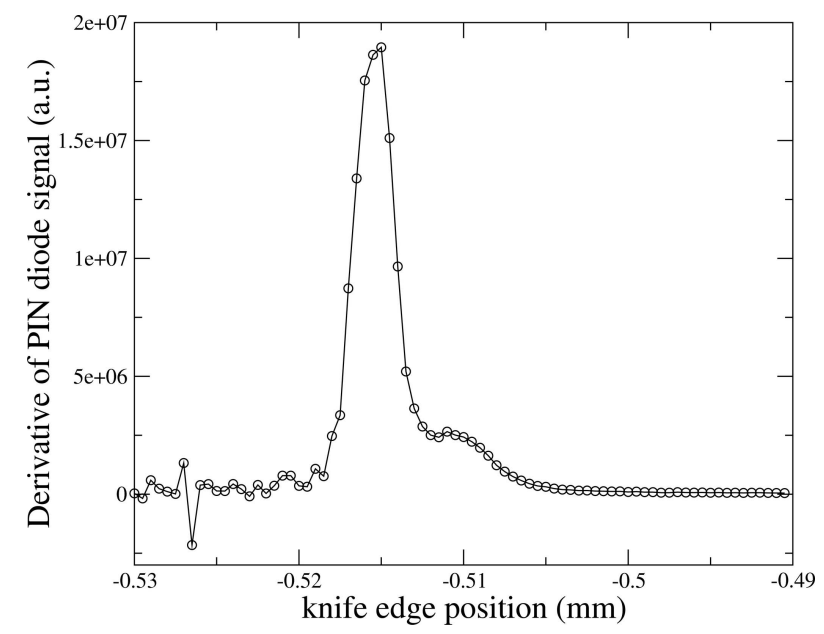

Figure 10

Vertical beam profile in the focal plane. diffracted by the white-beam slit diffracting half of the beam in 8-ID-E while the other half continued to 8-ID-I and reflected from the horizontally deflecting $\mathrm{Ge}(111)$ double-crystal monochromator. We recently reported how we changed the 8-ID-I monochromator from vertical to horizontal deflection (Kearney et al., 2018). The monochromator chamber was designed to accommodate either scattering direction (Narayanan et al., 2008). The speckle contrast at high angles in 8-ID-E was measured from a single crystal of $\mathrm{Fe}_{3} \mathrm{Al}$ at its antiphase domain superlattice peak with a wavevector of $1.89 \AA^{-1}$ (Brauer et al., 1995). In 8-ID-E, a PI LCX:1300 CCD camera with $20 \mu \mathrm{m}$ pixels was placed $1.1 \mathrm{~m}$ from the focal spot. Because the data in WA-XPCS have a diffuse peak with often arbitrary shape, the data were smoothed with a digital filtering technique developed earlier (Fluerasu et al., 2005). In this test, the 8-ID-I camera is a Medipix 3 Lambda 750k PAD from XSpectrum with $55 \mu \mathrm{m}$ pixels, placed $3.93 \mathrm{~m}$ downstream of the aerogel sample discussed earlier. The image distances $i$ are

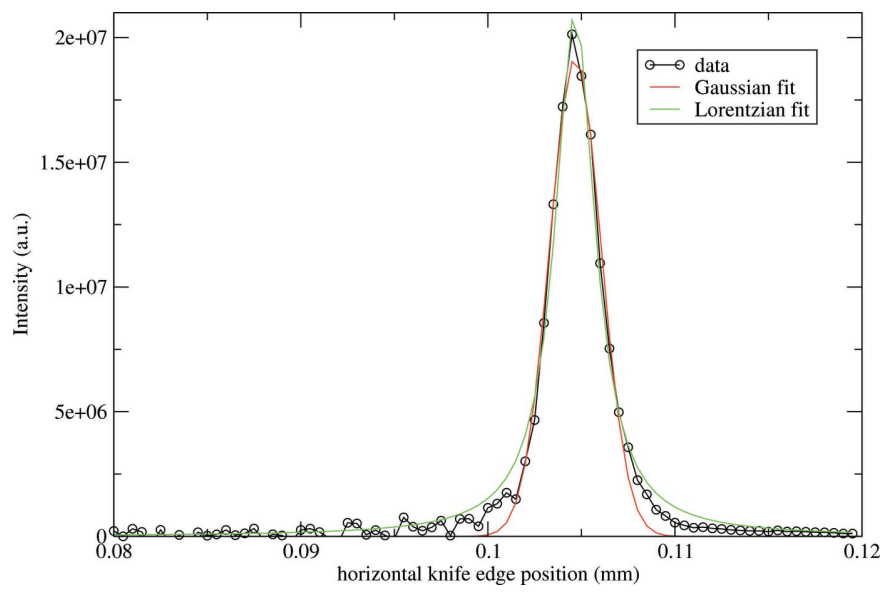

Figure 11

Horizontal beam profile in the focal plane from the derivative of the knife-edge scan, with a Gaussian and Lorentzian fit. 
for the horizontal and vertical direction, respectively. It uses the thin lens formula with the source at the white-beam slit position in the horizontal direction as stated earlier in Section 3. The calculated focal spot size $w$ is from equation (12). We found that we needed to reduce the horizontal beam acceptance to $100 \mu \mathrm{m}$ in 8-ID-I to produce the best focus. It is not clear why at this time. The results from the tests are shown in Table 2. The focal spot sizes compare well with the simple estimates provided in equation (12), particularly after correcting for the broadening from astigmatism using equation (14). It is not clear why the measured focal vertical spot in 8-ID-E differs from equation (14). We succeeded in focusing in 2D with round parabolic lenses on two beamlines, delivering good partially coherent flux and speckle contrast for XPCS experiments. This optical configuration was used for the remainder of 2019 at $7.35 \mathrm{keV}$ for $25 \%$ of the beam time on 8 -ID, with a partially coherent flux on the sample in 8-ID-E of $1.0 \pm 0.5 \times 10^{10}$ photons s $^{-1}$, with the observed variation caused by different upstream alignment conditions.

More recently we also tested the same optics in 8-ID-E with the same conditions as Table 2 but with a wide-open horizontal white-beam slit (see Appendix $C$ ). The condition results in a $2.8 \mu \mathrm{m}$ vertical spot size consistent with Table 2 , but the horizontal spot size is broader than the calculated image of the source or pinhole by 43 to $72 \%$, respectively, which further adds evidence of a heat bump also present on the water-cooled Si monochromator.

\section{Discussion}

Experimenters performing XPCS experiments typically define coherent flux as the partially coherent flux incident on their sample after an entrance aperture and some optics that produced speckle with a measurable contrast. The entrance aperture can be set to one coherent length or many, depending on the need for the experiment (Falus et al., 2006). For samples that are radiation sensitive, one prefers to illuminate the sample with a single transverse coherent mode to maximize the contrast (DeCaro et al., 2013).

Furthermore, the speckle contrast is maximum when a detector oversamples the speckle pattern, and starts to decline when the ratio of the speckle area to the pixel area here called $r_{\mathrm{s}}$ is less than unity (Dufresne et al., 2002; Falus et al., 2006). In the visible-light photon correlation spectroscopy community, it is well known that the signal-to-noise ratio of the time correlation function improves in fact and peaks at about $r_{\mathrm{s}} \simeq$ 0.1 for single speckle measurements (Schätzel, 1990). This scheme can be employed for samples that are insensitive to radiation damage (Dufresne et al., 2002). For samples that are radiation sensitive, one finds it best to match the speckle area to the pixel area, both to enhance the range of wavevector covered by a camera and to maximize the contrast which is a parameter in the signal-to-noise ratio (Falus et al., 2006; Vodnala et al., 2018).

Returning to our measurements performed near $11 \mathrm{keV}$, we measured a focused partially coherent flux of about $1.3 \times 10^{10}$ photons s$^{-1}$. This is a factor $30 \%$ higher than without lenses in a $20 \mu \mathrm{m} \times 20 \mu \mathrm{m}$ area and with a fully opened horizontal white-beam slit. Since $20 \mu \mathrm{m}$ is a typical coherence slit opening used at $7.35 \mathrm{keV}$, we should compare the flux measured in an area $(11 / 7.35)^{2}=2.2$ times smaller since the coherence lengths shrink by the ratio of energies. The partially coherent flux is thus about a factor $1.3 \times 2.2=$ 2.9 times higher than in unfocused conditions optimized for $11 \mathrm{keV}$. As noted in Table 1, the vertical opening in front of the lens could have been increased also to match the transverse coherence length in this direction (factor 1.8 possible). These tests were also performed with a single undulator A, but two inline phased undulators are available on the 8-ID straight section (factor 2.5 possible at $11 \mathrm{keV}$ ).

There is another valuable function of reducing the whitebeam slit opening in conditions where the optics cannot preserve the brightness of the source due to spatial strain induced by the absorbed power density on the surface of the optics (Antimonov et al., 2016). We reduced the total power on the first $\mathrm{Ge}(111)$ crystal of the double-crystal monochromator in 8-ID-I by a factor of seven with the smaller slit and were able to produce a diffraction-limited spot in the horizontal direction, and a much improved vertical focus on 8-ID-I. We note that these measurements were performed when the monochromator diffracted vertically (Kearney et al., 2018).

For samples that are not prone to radiation damage, 2D focusing may be an advantage to match the speckle size to the pixel size. The PI camera used in this work has a pixel size of $20 \mu \mathrm{m}$, thus we measured a speckle size of around $120-140 \mu \mathrm{m}, 4 \mathrm{~m}$ from the sample. This is fairly well matched to a modern PAD with pixel size ranging from 50 to $200 \mu \mathrm{m}$. Modern PADs absorb X-rays far more efficiently in their thick high- $Z$ sensor $(0.5 \mathrm{~mm})$ than deep depletion silicon CCDs do, thus focusing may be helpful also with a PAD to allow their use with moderate sample-detector distance in existing facilities at X-ray energies above $10 \mathrm{keV}$.

We did not explore thoroughly the reduction of parasitic scattering from slits, Be lens and windows in this setup. A single 2D slit before the lens produced the SAXS speckle patterns of the aerogel sample. We found this setup remarkably free from parasitic scattering. In an ultra-small-angle scattering experiment, we have noted some background from the larger incoming angular divergence, thus one uses guard slits to reduce the long tails on the focus. Although, since 2015, 2D focusing has only been used at $7.35 \mathrm{keV}$, one should be able to image the pinhole described earlier and placed upstream of the white-beam slit to produce higher intensity on samples at $11 \mathrm{keV}$ with some reduction in coherence. In light of recent evidence presented in Appendix $C$, one may require additional horizontal focusing to compensate for the heat bump of the Ge monochromator in 8-ID-I.

We note that this optical scheme could be helpful at other synchrotrons with horizontal source sizes comparable with or larger than the APS. Its use may also be helpful at highbrightness rings such as Petra III, NSLS-2, or the new multiple bend achromat sources (Tavares et al., 2018) when focusing high-energy X-rays above $20 \mathrm{keV}$. A high-precision and stable slit is needed for this application. 


\section{Conclusions}

This paper demonstrated the use of a white-beam slit to spatially filter the horizontal coherence and focus the beam in two dimensions with refractive lenses, producing improvements in partially coherent flux and higher focal plane intensities on samples. Our water-cooled optics also benefit from the power reduction on the monochromators. The scheme is in routine use at two 8-ID beamlines sharing a single in-line straight section where two simultaneous XPCS experiments can be performed.

\section{APPENDIX $A$}

Horizontal beam profile at the lens with a small white-beam slit opening

If we assume that the irradiance from a horizontal infinitesimal portion of the source at position and angular emission angle $\left(x, x^{\prime}\right)$ is Gaussian in both spatial and angular coordinates, then the intensity generated by this element is

$$
I\left(x, x^{\prime}\right) \propto \exp \left\{-0.5\left[x^{2} / \Sigma_{x}^{2}+\left(x^{\prime} / \Sigma_{x^{\prime}}\right)^{2}\right]\right\} \text {. }
$$

When we close the white-beam slit to an opening $\Delta$, we image the source with a pinhole camera. Ignoring diffraction effects, the intensity at position $x_{1}$ in the lens plane is an image of the source irradiance with

$$
\begin{aligned}
& x^{\prime}=x_{1} /\left(R_{1}-R_{\mathrm{s}}\right) \quad \text { and } \\
& x=R_{\mathrm{s}} x^{\prime}=x_{1} R_{\mathrm{s}} /\left(R_{1}-R_{\mathrm{s}}\right) .
\end{aligned}
$$

Replacing $x$, and $x^{\prime}$ in equation (16), we find that the beam profile in the plane of the lens is

$$
\begin{aligned}
I\left(x_{1}\right)= & I_{0} \exp \left(-0.5\left\{\left[\frac{x_{1} R_{\mathrm{s}}}{\Sigma_{x}\left(R_{1}-R_{\mathrm{s}}\right)}\right]^{2}\right.\right. \\
& \left.\left.+\left[\frac{x_{1}}{\Sigma_{x^{\prime}}\left(R_{1}-R_{\mathrm{s}}\right)}\right]^{2}\right\}\right),
\end{aligned}
$$

and thus a Gaussian in coordinate $x_{1}$ depending on both RMS source size and divergence, with a RMS beam size given in equation (8).

\section{APPENDIX $B$}

\section{Speckle metrology}

A typical pattern from an aerogel measured with the PI camera is shown in Fig. 12. The intensity is displayed with a color map from blue (low intensity) to red (highest intensity).

A standard technique to estimate the speckle size computes the 2D spatial autocorrelation function of the speckle pattern (Abernathy et al., 1998; Tsui et al., 1998; Sandy et al., 1999). To remove the well known contribution due to Poisson noise of the detector (Dufresne et al., 1995), we perform a time average of a cross-correlation function of two speckle patterns followed in time by one exposure period. Here, 63 such crosscorrelation functions were averaged, and the overall impact on

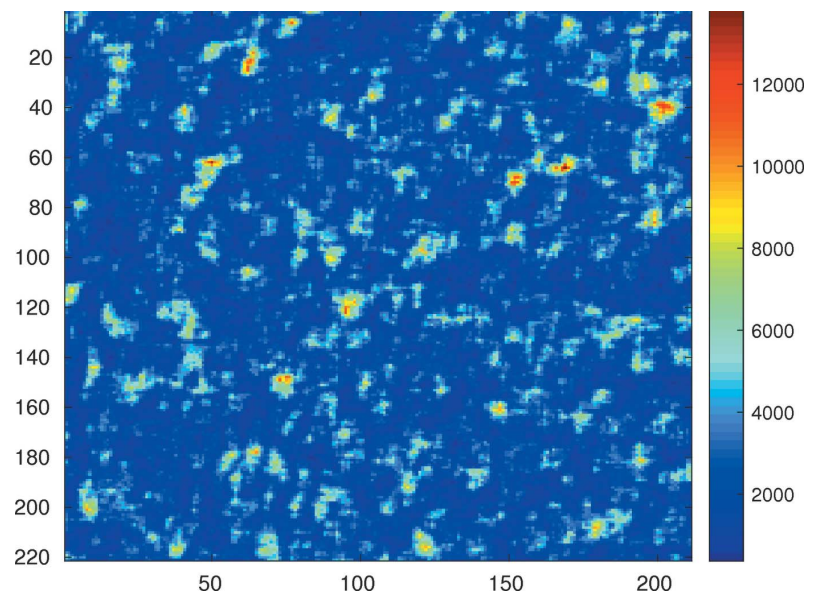

Figure 12

2D speckle pattern of the aerogel measured at $10.63 \mathrm{keV}$, with a PI camera with $20 \mu \mathrm{m}$ pixels, $4 \mathrm{~m}$ from the sample. The color scale is displayed in analog-to-digital units for this 16-bit camera.

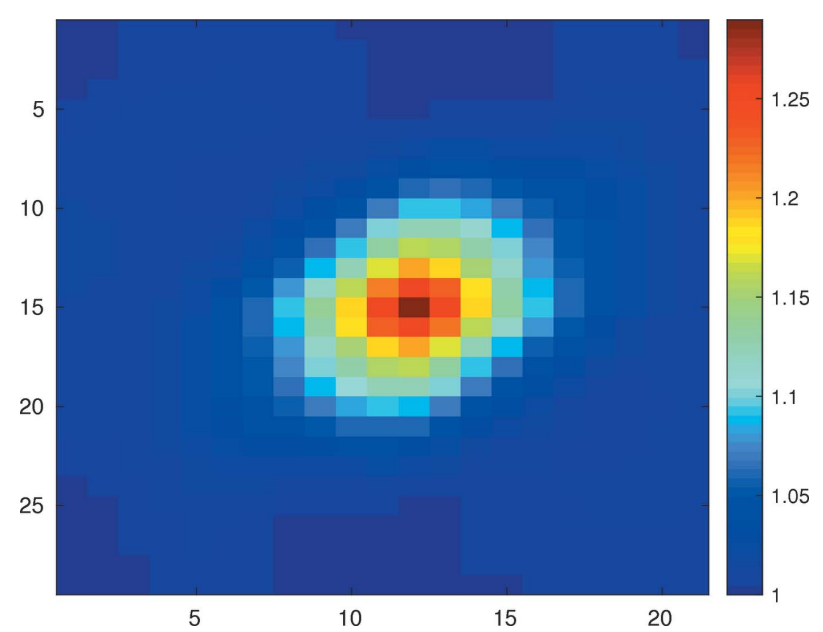

Figure 13

2D spatial autocorrelation function of a speckle pattern measured at $10.63 \mathrm{keV}$. The horizontal and vertical scales are in units of pixels.

these measurements reduces the peak by 0.01 . The crosscorrelation function data are normalized to unity at large spatial lag.

A typical spatial cross-correlation function is shown in Fig. 13. Cuts in both directions were fit to Gaussians. A horizontal cut of Fig. 13 is shown in Fig. 14 with its Gaussian fit. The contrast is the peak value of the autocorrelation function and is slightly higher than the value obtained from the speckle size fit shown as a legend. The resulting FWHM in both direction were plotted in Fig. 8 .

\section{APPENDIX C}

Beam profiles in 8-ID-E with a wide open horizontal white-beam slit

In July 2020, we performed a focusing test with the same lenses in 8-ID-E with a wide open horizontal white-beam slit. 


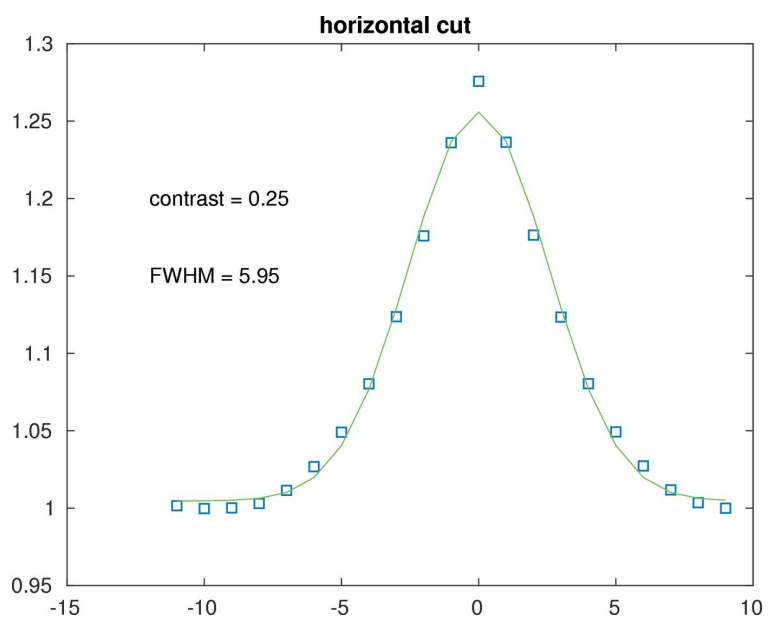

Figure 14

An horizontal slice of the 2D spatial autocorrelation function in Fig. 13. The solid line is a least-square fit to a Gaussian. The horizontal axis is in units of pixels.

The conditions were the same as Table 2. Fig. 15 shows two beam profiles probed with the knife-edge, the sharp focus is in the vertical, while the broad one is in the horizontal. The fits are arctan fits, the FWHMs quoted are from the derivative of the fits. The vertical beam FWHM derived from the fit is $2.8 \mu \mathrm{m}$. One would expect to either demagnify the horizontal source $(20 \mu \mathrm{m}$ FWHM) or the pinhole $(16.6 \mu \mathrm{m}$ FWHM). We observe $28.6 \mu \mathrm{m}$ FWHM horizontally. We suspect this broadening of the focal spot is caused by the heat bump of the $\mathrm{Si}$ monochromator which is not observed in the vertical. The flux was $4.35 \times 10^{10}$ photons $^{-1}$ in this mode, while the following week it was $7.5 \times 10^{9}$ photons $\mathrm{s}^{-1}$ with the WB slit closed to $40 \mu \mathrm{m}$, a factor of 5.8 higher. The ratio of the pinhole and the WB slit opening is 6.9 , thus the flux is $18 \%$ lower than expected. This condition would provide very low contrast for XPCS, i.e. over ten coherence mode horizontally. These measurements complement our initial observation in 8-ID-I.

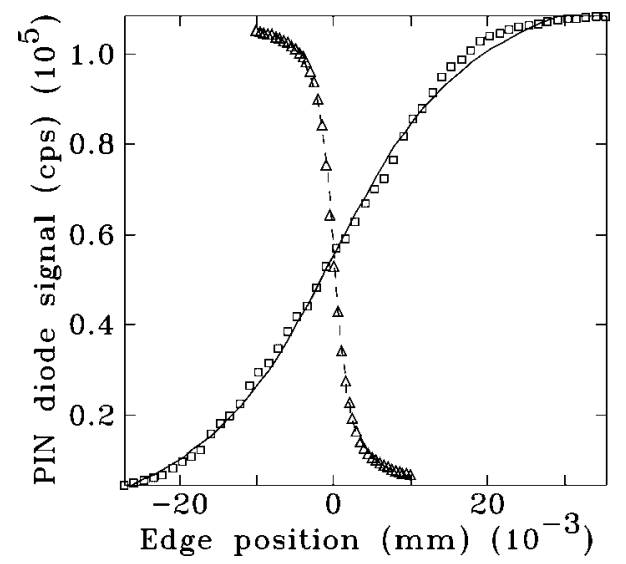

Figure 15

Horizontal (squares) and vertical (triangles) knife-edge scans of the beam profile in 8-ID-E. The solid and dashed lines are least-squares fit discussed in the text.

\section{Acknowledgements}

We wish to thank Michael Fisher for the design of the lens holder, and Ray Ziegler for technical support. We are grateful for helpful discussions with Zhang Jiang, Oliver Schmidt, and Deming Shu. This material is based upon work supported by Laboratory Directed Research and Development (LDRD) funding from Argonne National Laboratory, provided by the Director, Office of Science, of the US Department of Energy under Contract No. DE-AC02-06CH11357. This research was performed on beamline 8-ID-I of the Advanced Photon Source, a US Department of Energy (DOE) Office of Science User Facility operated for the DOE Office of Science by Argonne National Laboratory under Contract No. DE-AC0206 CH11357.

\section{Funding information}

Funding for this research was provided by: US Department of Energy Office of Science User Facility (award No. DE-AC0206CH11357).

\section{References}

Abernathy, D. L., Grübel, G., Brauer, S., McNulty, I., Stephenson, G. B., Mochrie, S. G. J., Sandy, A. R., Mulders, N. \& Sutton, M. (1998). J. Synchrotron Rad. 5, 37-47.

Antimonov, M., Khounsary, A., Sandy, A., Narayanan, S. \& Navrotski, G. (2016). Nucl. Instrum. Methods Phys. Res. A, 820, 164-171.

Brauer, S., Stephenson, G., Sutton, M., Brüning, R., Dufresne, E., Mochrie, S., Grübel, G., Als-Nielsen, J. \& Abernathy, D. (1995). Phys. Rev. Lett. 74, 2010-2013.

Chsuhkin, Y. (2020). ID10 EH2 Beamline Description. Technical Report. European Synchrotron Radiation Facility, Grenoble, France.

Chubar, O., Fluerasu, A., Berman, L., Kaznatcheev, K. \& Wiegart, L. (2013). J. Phys. Conf. Ser. 425, 162001.

DeCaro, C., Karunaratne, V. N., Bera, S., Lurio, L. B., Sandy, A. R., Narayanan, S., Sutton, M., Winans, J., Duffin, K., Lehuta, J. \& Karonis, N. (2013). J. Synchrotron Rad. 20, 332-338.

Dierker, S. (1997). In Light Scattering and Photon Correlation Spectroscopy, Proceedings of a NATO Advanced Research Workshop, edited by E. Pike. Dordrecht: Kluwer Academic.

Dufresne, E., Brüning, R., Sutton, M., Rodricks, B. \& Stephenson, G. B. (1995). Nucl. Instrum. Methods Phys. Res. A, 364, 380393.

Dufresne, E. M., Dierker, S. B., Yin, Z. \& Berman, L. (2009). J. Synchrotron Rad. 16, 358-367.

Dufresne, E. M., Nurushev, T. S., Clarke, R. \& Dierker, S. B. (2002). Phys. Rev. E, 65, 061507.

Elleaume, P. (2002). Undulators, Wigglers and Their Applications, edited by H. Onuki \& P. Elleaume, p. 79. London: Taylor and Francis.

Falus, P., Lurio, L. B. \& Mochrie, S. G. J. (2006). J. Synchrotron Rad. 13, 253-259.

Fluerasu, A., Sutton, M. \& Dufresne, E. M. (2005). Phys. Rev. Lett. 94, 055501.

Goodman, J. (1985). Statistical Optics, 1st ed. New York: John Wiley \& Sons.

Kearney, S., Dufresne, E., Narayanan, S., Sandy, A. \& Shu, D. (2018). Proceedings of the 10th Mechanical Engineering Design of Synchrotron Radiation Equipment and Instrumentation 
(MEDSI'18), 25-29 June 2018, Paris, France, pp. 391-393. Geneva: JACoW Publishing.

Lengeler, B., Schroer, C. G., Benner, B., Gerhardus, A., Günzler, T. F., Kuhlmann, M., Meyer, J. \& Zimprich, C. (2002). J. Synchrotron Rad. 9, 119-124.

Libbert, J. L., Pitney, J. A. \& Robinson, I. K. (1997). J. Synchrotron Rad. 4, 125-127.

Livet, F. \& Sutton, M. (2012). C. R. Phys. 13, 227-236.

Narayanan, S., Sandy, A., Shu, D., Sprung, M., Preissner, C. \& Sullivan, J. (2008). J. Synchrotron Rad. 15, 12-18.

Sandy, A. R., Lurio, L. B., Mochrie, S. G. J., Malik, A., Stephenson, G. B., Pelletier, J. F. \& Sutton, M. (1999). J. Synchrotron Rad. 6, 1174-1184.

Sandy, A. R., Narayanan, S., Sprung, M., Su, J.-D., Evans-Lutterodt, K., Isakovic, A. F. \& Stein, A. (2010). J. Synchrotron Rad. 17, 314320.

Sandy, A. R., Zhang, Q. \& Lurio, L. B. (2018). Annu. Rev. Mater. Res. 48, 167-190.

Schätzel, K. (1990). Quantum Opt. 2, 287-305.

Schroer, C. G. \& Falkenberg, G. (2014). J. Synchrotron Rad. 21, 9961005.

Shi, X., Reininger, R., Sanchez del Rio, M. \& Assoufid, L. (2014). J. Synchrotron Rad. 21, 669-678.

Sikorski, M., Song, S., Schropp, A., Seiboth, F., Feng, Y., AlonsoMori, R., Chollet, M., Lemke, H. T., Sokaras, D., Weng, T.-C., Zhang, W., Robert, A. \& Zhu, D. (2015). J. Synchrotron Rad. 22, 599-605.

Singer, A. \& Vartanyants, I. A. (2014). J. Synchrotron Rad. 21, 5-15.
Sinha, S. K., Jiang, Z. \& Lurio, L. B. (2014). Adv. Mater. 26, 7764 7785.

Snigirev, A., Kohn, V., Snigireva, A. \& Lengeler, B. (1996). Nature, 384, 49-51.

Sutton, M. (2008). C. R. Phys. 9, 657-667.

Tavares, P. F., Al-Dmour, E., Andersson, Å., Cullinan, F., Jensen, B. N., Olsson, D., Olsson, D. K., Sjöström, M., Tarawneh, H., Thorin, S. \& Vorozhtsov, A. (2018). J. Synchrotron Rad. 25, 1291-1316.

Tsui, O. K. C., Mochrie, S. G. J. \& Berman, L. E. (1998). J. Synchrotron Rad. 5, 30-36.

Vodnala, P., Karunaratne, N., Lurio, L., Thurston, G. M., Vega, M., Gaillard, E., Narayanan, S., Sandy, A., Zhang, Q., Dufresne, E. M., Foffi, G., Grybos, P., Kmon, P., Maj, P. \& Szczygiel, R. (2018). Phys. Rev. E, 97, 020601.

Wiegart, L., Rakitin, M., Zhang, Y., Fluerasu, A. \& Chubar, O. (2019). AIP Conf. Proc. 2054, 060079.

Winarski, R. P., Holt, M. V., Rose, V., Fuesz, P., Carbaugh, D., Benson, C., Shu, D., Kline, D., Stephenson, G. B., McNulty, I. \& Maser, J. (2012). J. Synchrotron Rad. 19, 1056-1060.

Yang, B., Lee, S., Morgan, J. \& Shang, H. (2017). Proceedings of the 5th International Beam Instrumentation Conference (IBIC 2016), Barcelona, Spain, edited by I. Costa, U. Iriso, F. Pérez \& V. R. Schaa, pp. 504-507. JACoW.

Zhang, Q., Dufresne, E. M., Grybos, P., Kmon, P., Maj, P., Narayanan, S., Deptuch, G. W., Szczygiel, R. \& Sandy, A. (2016). J. Synchrotron Rad. 23, 679-684.

Zozulya, A. V., Bondarenko, S., Schavkan, A., Westermeier, F., Grübel, G. \& Sprung, M. (2012). Opt. Express, 20, 18967-18976. 